\title{
Rupture of a Healthy Uterus during the 2nd Quarter of Pregnancy about a Case and Review of Literature
}

\author{
Berrada $\mathrm{T}^{*}$, Lakhdar A, Zeraidi N, Baidada A \\ Gynecology Obstetrics and Gynecological Endoscopy Maternity Souissi Rabat Morocco
}

DOI: $10.36348 /$ sijog.2020.v03i07.002

| Received: 18.06.2020 | Accepted: 25.06.2020 | Published: 21.07 .2020

*Corresponding author: Dr. Berrada Taher

Abstract

Uterine rupture on healthy uterus is a very rare obstetric complication; few cases have been described in the literature. We report a case of rupture taken care of in our training. Multiparity and cross-presentation were the main risk factors. The treatment was conservative with a tubal section tightening to avoid the risk of recurrence.

Keywords: Pregnancy, healthy uterus, uterine rupture.

Copyright @ 2020: This is an open-access article distributed under the terms of the Creative Commons Attribution license which permits unrestricted use, distribution, and reproduction in any medium for non-commercial use (NonCommercial, or CC-BY-NC) provided the original author and source are credited.

\section{INTRODUCTION}

Uterine rupture on a healthy uterus is a rare obstetric complication, involving vital maternal and fetal prognoses and obstetric outcome in the absence of immediate treatment. We report a case of spontaneous uterine rupture on no-cicatricial uterus in our maternity ward. We discuss the risk factors, the signs of clinical calls and the different therapeutic modalities that can be implemented, based on an observation and a review of the literature.

\section{CASE}

Patient 31 years old, without significant pathological antecedents, 5th gestures 4th pares with 3 live children, all the pregnancies are carried out vaginally. The 5th gesture is the current pregnancy estimated at 32 weeks +3 days according to the date of the last period. Admitted four days ago for the threat of premature labor with premature rupture of the membranes. Examination at admission: $68 \mathrm{~kg}$ weight, height $1.69 \mathrm{~m}$, blood pressure $11 / 7 \mathrm{cmHg}$, temperature $37^{\circ} \mathrm{C}$, uterine height at $24 \mathrm{~cm}$, fetal heart sound was present regular. The vaginal touch found a soft, half long neck, admits 2 fingers, empty excavation, pocket of clear liquid broken water. Obstetrical ultrasound showed a progressive mono-fetal pregnancy, positive cardiac activity, transverse presentation, biparietal diameter at $80 \mathrm{~mm}$ and femoral length at $65 \mathrm{~mm}$, which corresponded to the measurement of 32-33 weeks of amenorrhea. An assessment was made of the presence of iron deficiency hemoglobin at $8.9 \mathrm{~g} / \mathrm{dl}$, thrombocytopenia at 123,000/ $\mathrm{mm} 3$ and CRP at 20. The cytobacteriological examination of the urine and the vaginal sampling were without particularities. The patient was tocolysed with nifedipine $20 \mathrm{mg}$ every 8 hours, antibiotic prophylaxis amoxicillin one gram every 8 hours. Fetal heart rate recording was oscillating and responsive with a baseline rhythm of 130 beats per minute. During the surveillance of our guard the patient presented uterine contracture with blackish bleeding, fetal heart sounds were present, caesarean section was indicated for suspicion of retroplacental hematoma with positive fetal heart sounds which allowed the Podalic extraction of a new male Apgar 10/10 birth weight $1890 \mathrm{~g}$. the hematoma occupied $50 \%$ of the placenta. At the exploration presence of a corporal rupture at the posterior surface of the uterus of $5 \mathrm{~cm}$ (fig1), it was taken up by a wire resobable in two planes. Tubal section ligation was performed. Hemostasis was ensured, the patient did not have uterine inertia. Valve examination of the genital spine did not reveal the presence of a cervical or vaginal tear. The postoperative blood count showed anemia at $5.3 \mathrm{~g} / \mathrm{dl}$, which required transfusion of 2 red blood cells. The patient left the hospital on the 5 th day of the postpartum.

\section{DISCUSSION}

Complete uterine rupture is a solution of continuity affecting the uterine wall, as well as visceral 
peritoneum that may occur spontaneously or traumatically, following a shock, an obstetric or instrumental maneuver. This generates communication between the uterus and the peritoneal cavity. The frequency in France is estimated between 1/17000 and $1 / 20000$ deliveries [1]. In developing countries, the frequency is higher because of the poor surveillance facilities [2].

The factors favoring non-cicatricial uterine rupture of the uterus according to the literature are [2-4]:

- Multiparity, responsible for myometrium fragility, our patient was a 4 th par.

- Fetal macrosomia.

- The type of presentation: the presentation of the seat and the transversal presentation. Our case was in transverse presentation.

- The misuse of oxytocic.

- The use of obstetric maneuvers or instrumental extractions.

- Other etiological factors: fetopelvic disproportions, uterine expressions during vaginal delivery, placental insertion abnormalities (mainly placenta accreta and percreta), curettage, hysteroscopic resections and myomectomy history.

Clinically, the association of acute abdominal pain, uterine hypertonia, signs of shock, metrorrhagia, gross hematuria and impaired fetal heart rate are clinically described, but inconsistently found in their totality [5]. Our patient had abdominal pain with clinical signs of a retroc placental hematoma with present fetal heart sounds, hence a caesarean section was indicated. However, retro-placental hematoma is the main differential diagnosis of uterine rupture [3].

On an anatomo-pathological level, the uterine rupture occurring during the work sit favorably at the level of the lower segment, whereas those which take place outside the work are corporeal, a cervical or vaginal lesion is always to look for in case of uterine rupture. outside of labor by valve examination [6].

Uterine rupture can occur at any time during pregnancy, but mainly in the 2nd trimester and during labor. During the 2nd trimester uterine rupture occurs in particular situations: cornual or interstitial pregnancies, history of perforation, pregnancy on caesarean section scar ... [5].

The management of uterine rupture remains a medical and surgical emergency. The severity of maternal-fetal consequences is partly related to response time. It requires measures of immediate resuscitation of shock and correction of hemodynamic disorders. The choice of treatment (hysterectomy or conservative treatment) depends on the anatomical lesions observed. Hysterectomy was the gold standard for complete rupture. Data from the literature have shown the possibility of conservative treatment with complete uterine rupture repair should be undertaken when technically feasible. We were satisfied with conservative treatment since the lesion was surgically accessible and haemostasis was ensured. Tubal section ligation was performed because of multiparity and the risk of recurrence [2,5].

Uterine rupture of the uterus without scarring is an emergency with maternal and fetal prognosis. Ahmadi et al [2] found in their studies two cases of maternal deaths with seven fetal deaths perpartum. Thus, in other series in the literature, a percentage of $14 \%$ to $20 \%$ of maternal deaths and $40 \%$ to $60 \%$ of fetal deaths. Uterine ruptures on non-scarred uterus have a higher percentage of maternal deaths than those on a scarred uterus.

\section{CONCLUSION}

Uterine rupture on a healthy uterus is a rare obstetric complication. The maternal and fetal vital prognosis is at stake. Surgical management is the cornerstone of treatment.

\section{REFERENCE}

1. Fatfouta, S., Villeroy de Galhau, J., Dietsch, E., Eicher, D. (2008). Perrin Spontaneous uterine rupture on healthy uterus during labor: about a case and review of the literature Journal of Gynecology Obst And Biology of Reproduction, 37, 200-203

2. Ahmadi, M., Nouira, M., Bibi, S., Boughuizane, H., Saidi, A., Chaib, H., Khairi. (2003). Uterine rupture on healthy gravid uterus. About 28 cases. Gynecology Obstetrics \& Fertility, 31, 713-717

3. Walsh, C. A., \& Baxi, L. V. (2007). Rupture of the primigravid uterus: a review of the literature. Obstetrical \& gynecological survey, 62(5), 327-334.

4. Sakr, R., Berkane, N., Barranger, E., Dubernard, G., Darai, E., \& Uzan, S. (2007). Unscarred uterine rupture--case report and literature review. Clinical and experimental obstetrics \& gynecology, 34(3), 190-192.

5. Parant, O. (2012). Uterine rupture: prediction, diagnosis et management. Journal de gynecologie, obstetrique et biologie de la reproduction, 41(8), 803-816. 1

6. Schrinsky, D. C., \& Benson, R. C. (1978). Rupture of the pregnant uterus: a review. Obstetrical \& gynecological survey, 33(4), 217-232. 